\title{
Life-Threatening Complications of Endotracheal Stenting Caused by Respiratory Secretions
}

\author{
Sooim Shin, Ye Jin Lee, Hye Rin Kang, Jin Hwa Song, Young Sik Park \\ Department of Internal Medicine, Seoul National University Hospital, Seoul, Korea
}

\begin{abstract}
Airway stenting has become a common treatment for malignant central airway obstruction. Most airway stents are well tolerated, and life-threatening complications are very rare. Here we describe the case of a life-threatening obstruction due to accumulated respiratory secretions. A 33-year-old man had a massive large cell neuroendocrine carcinoma in the anterior mediastinum, which caused extensive extrinsic compression of the whole trachea. Airway stents were successfully inserted into the whole trachea but 1 week after the procedure, the patient complained of worsening dyspnea due to sticky respiratory secretions accumulated inside the stents. Because the patient could not expectorate a large amount of sticky secretions, frequent bronchoscopic toiletings were performed for 2 months. The stent was well maintained for 7 months till the enlarged tumor causes extrinsic compression because stent revision was performed. About 1 year after the first stent insertion, the patient was admitted to the intensive care unit and intubated because of obstruction of the stent by organized secretions. Fortunately, these secretions were successfully removed and the patient was discharged without any sequelae. Thus, respiratory secretions can cause life-threatening complications after airway stent insertion. Clinicians should be aware of this, and careful examination and close follow-up of such patients are needed.
\end{abstract}

Keywords: Self expandable metallic stents; Sputum; Complications; Morbidity

\section{INTRODUCTION}

Airway stenting is a common technique in malignant central airway obstruction for dyspnea [1-4]. The effectiveness of airway stenting in improving quality of life and resolving dyspnea has been proven $[1,2,4-9]$, and it has been reported to be technically successful in $90 \%$ of cases [9]. The possible complications of airway stenting are growth of granulation tissue, accumulated secretions, fracture or migration of the stent, airway wall perforation, and infection [1,2,7,10-13]. There is a lack of reports covering lifethreatening secretions caused by this procedure; here, we report the case in which a patient experienced life-threatening complications induced by accumulated secretions after airway stenting.

\section{CASE REPORT}

A 33-year-old man was referred to the respiratory clinic of Seoul National University Hospital because of resting dyspnea. The patient had been diagnosed with a massive large cell neuroendocrine carcinoma in the anterior mediastinum 2 years previously, and he had been receiving chemotherapy and radiation treatment. However, the tumor progressed in the anterior mediastinum, causing extensive extrinsic compression of the whole trachea (Fig. 1).

Urgent airway stent insertion was performed under general anesthesia using a rigid bronchoscope. Two silicon-covered metal stents (Hercules Airway model no. AW-10-40; S\&G BIOTECH Inc., Seongnam, Korea) were successfully deployed in the whole trachea, and dyspnea was immediately relieved (Fig. 2). However, 1 week after inserting the stents, the patient complained of worsening dyspnea, cough, and sticky respiratory secretions. Subse-
Correspondence to: Young Sik Park

Department of Internal Medicine, Seoul National University Hospital, 101 Daehak-ro, Jongno-gu, Seoul 03080, Korea

Tel: +82-2-2072-7214, Fax: +82-2-762-9662, E-mail: mdyspark@gmail.com

Received: Jul. 22, 2016 / Accepted after revision: Sep. 6, 2016
(C) 2016 Soonchunhyang Medical Research Institute This is an Open Access article distributed under the terms of the Creative Commons Attribution Non-Commercial License (http://creativecommons.org/licenses/by-nc/4.0/) 

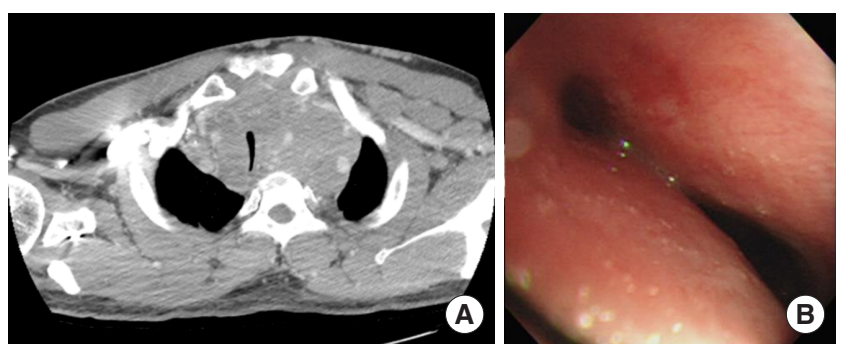

Fig. 1. Malignant central airway obstruction in the patient. (A) Chest computed tomography and (B) bronchoscopy show extensive extrinsic obstruction of the whole trachea.
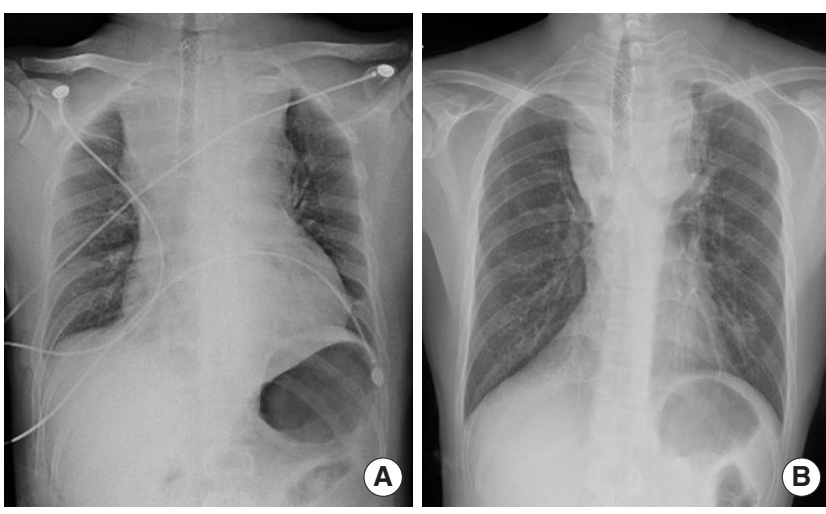

Fig. 2. (A, B) Chest radiograph revealing a patent trachea immediately after stent insertion.

quently, bronchoscopy revealed impending obstruction of the stents by accumulation of the very sticky secretions (Fig. 3). Frequent bronchoscopic toileting was therefore required, and this complication was well-controlled for several months. After 7 months, the carina gradually became compressed; therefore, silicon Y-stent revision was performed. After stent revision, regular bronchoscopic toileting was still required for the accumulated sticky secretions.

Unfortunately, about 4 months after inserting the Y stent, he was admitted to the intensive care unit (ICU) for intubation, as the respiratory secretions had become life-threatening. Dense, thick respiratory secretions obstructing the end of the stent were detected upon bronchoscopy (Fig. 4). Emergency bronchoscopic removal of the secretions was performed in the ICU, and the patient was discharged without any sequelae.

\section{DISCUSSION}

It is inevitable that inserting stents in the airway triggers inflammatory reactions. Generation of massive secretions after air-
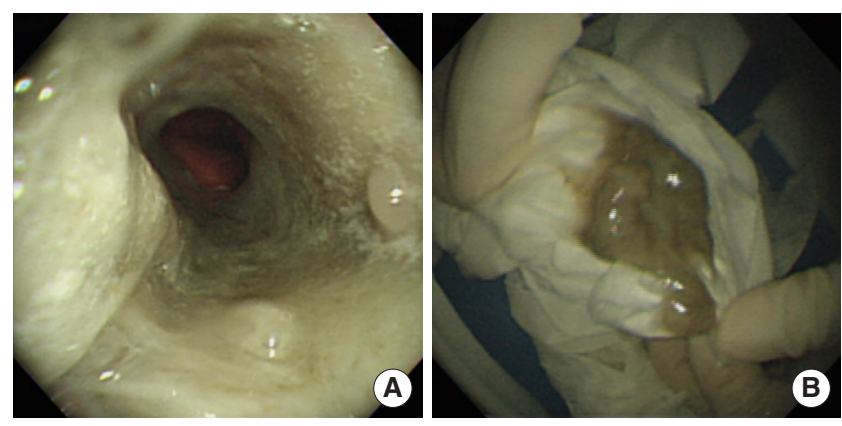

Fig. 3. (A, B) Impending obstruction of the stents by accumulated secretions.

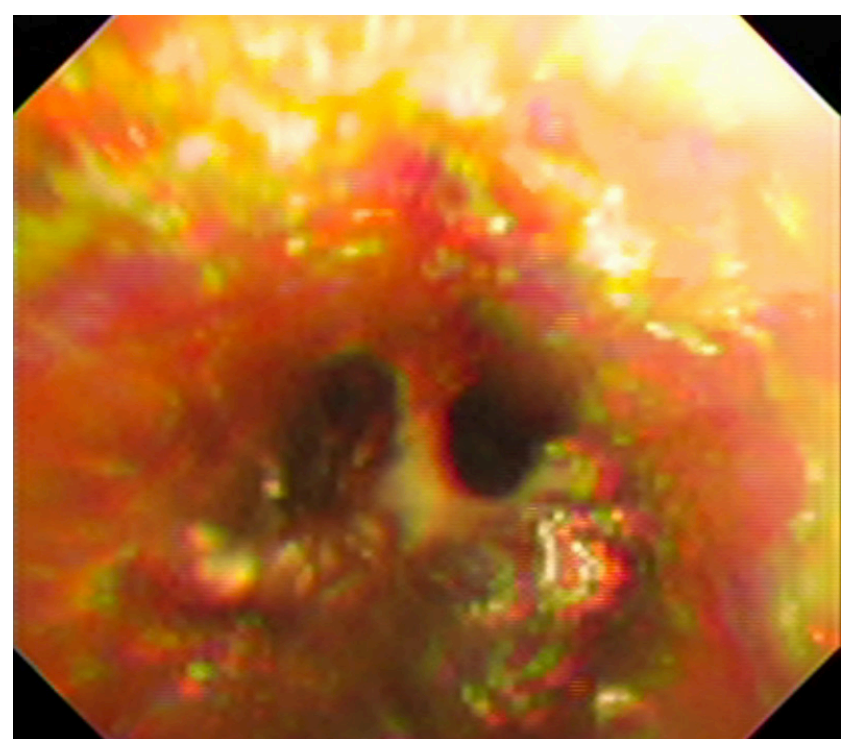

Fig. 4. Life-threatening accumulated secretions.

way stent insertion is caused by airway irritation of the foreign body, and the retention of mucous secretions is caused by impaired mucociliary clearance $[4,14]$. Although secretions are known to be a common complication after inserting airway stents, there have been no reports covering a threat to life caused by secretions after inserting airway stents.

Previous studies dealing with life-threatening complications of airway stent insertion have included airway perforation, severe infection, bleeding, stent migration, and airway obstruction induced by granulation tissue. Secretions are considered to be a common complication, but not to be a serious complication $[1,14]$. Excessive retention of secretions is known to occur in $10 \%$ to $40 \%$ of cases [1$3,11,14]$. Numerous studies found that excessive secretion is prone to occur in silicone stent than metallic stent. This is attributable to the mesh frame of metallic stents, which permits mucociliary clearance more easily $[1,2,11,14,15]$. In our experience, secretions, 
regardless of severity, occur unexceptionally so that clinicians have to pay attention to every case.

As the use of airway stents increases, maintaining the stents has become increasingly important. Appropriate management and follow-up are essential for long-term maintenance. As we noted in this case, short-term bronchoscopic follow-up is indispensable for evaluating the amount and consistency of secretions. Thereafter, regular bronchoscopy can be performed if the secretions are found to be particularly thick and copious in the first bronchoscopy. Adequate hydration and mucolytics should be considered, and if needed, application of saline or mucolytics by nebulizer can also be used. Most importantly, physicians should constantly follow-up on symptoms and perform careful physical examination. Additionally, timely antibiotics and infection control are occasionally needed, because infection is directly associated with secretions. In severe cases, regular bronchoscopic toileting should be performed for extended periods. Our report is significant in that it presents an approach to optimal management for this life-threatening condition.

In conclusion, physicians should be aware of possible life-threatening complications due to secretions after insertion of airway stents. Further attention should now be given to preserving these stents from secretions, as this complication could be overcome by appropriate post-procedural management.

\section{REFERENCES}

1. Saji H, Furukawa K, Tsutsui H, Tsuboi M, Ichinose S, Usuda J, et al. Outcomes of airway stenting for advanced lung cancer with central airway obstruction. Interact Cardiovasc Thorac Surg 2010;11:425-8.
2. Ranu H, Madden BP. Endobronchial stenting in the management of large airway pathology. Postgrad Med J 2009;85:682-7.

3. Cavaliere S, Venuta F, Foccoli P, Toninelli C, La Face B. Endoscopic treatment of malignant airway obstructions in 2,008 patients. Chest 1996;110: 1536-42.

4. Filler RM, Forte V, Chait P. Tracheobronchial stenting for the treatment of airway obstruction. J Pediatr Surg 1998;33:304-11.

5. Razi SS, Lebovics RS, Schwartz G, Sancheti M, Belsley S, Connery CP, et al. Timely airway stenting improves survival in patients with malignant central airway obstruction. Ann Thorac Surg 2010;90:1088-93.

6. Chin CS, Litle V, Yun J, Weiser T, Swanson SJ. Airway stents. Ann Thorac Surg 2008;85:S792-6.

7. Furukawa K, Ishida J, Yamaguchi G, Usuda J, Tsutsui H, Saito M, et al. The role of airway stent placement in the management of tracheobronchial stenosis caused by inoperable advanced lung cancer. Surg Today 2010;40:315-20.

8. Amjadi K, Voduc N, Cruysberghs Y, Lemmens R, Fergusson DA, Doucette $\mathrm{S}$, et al. Impact of interventional bronchoscopy on quality of life in malignant airway obstruction. Respiration 2008;76:421-8.

9. Ost DE, Ernst A, Grosu HB, Lei X, Diaz-Mendoza J, Slade M, et al. Therapeutic bronchoscopy for malignant central airway obstruction: success rates and impact on dyspnea and quality of life. Chest 2015;147:1282-98.

10. Chhajed PN, Somandin S, Baty F, Mehta AJ, Azzola A, Leuppi J, et al. Therapeutic bronchoscopy for malignant airway stenoses: choice of modality and survival. J Cancer Res Ther 2010;6:204-9.

11. Lemaire A, Burfeind WR, Toloza E, Balderson S, Petersen RP, Harpole $\mathrm{DH} \mathrm{Jr}$, et al. Outcomes of tracheobronchial stents in patients with malignant airway disease. Ann Thorac Surg 2005;80:434-7.

12. Lee P, Kupeli E, Mehta AC. Airway stents. Clin Chest Med 2010;31:14150 .

13. Ost DE, Shah AM, Lei X, Godoy MC, Jimenez CA, Eapen GA, et al. Respiratory infections increase the risk of granulation tissue formation following airway stenting in patients with malignant airway obstruction. Chest 2012;141:1473-81.

14. Zakaluzny SA, Lane JD, Mair EA. Complications of tracheobronchial airway stents. Otolaryngol Head Neck Surg 2003;128:478-88.

15. Dasgupta A, Mehta AC. Use of wallstents in central airway obstruction. Oper Tech Otolaryngol Head Neck Surg 1999;10:264-70. 